\title{
NBP-IWT-DCT-SVD-PSO Based Secure Image Watermarking
}

\author{
CH. Ravi Kumar, R. Surya PrakasaRao, P. Rajesh Kumar
}

\begin{abstract}
This paper proposes energetic photograph watermarking approach contingent upon entire wide variety Wavelet alternate, Discrete cosine change, unique well really worth Disintegration and Molecule Swarm Streamlining. PSO is implemented to get an extremely good watermarking predictable. further, to make the watermarked picture continually fiery to disenchanted and flipping assaults, in advance than introducing the Standardized square system is achieved to the host image. because the population duration assembles, GA cannot overhaul the gadget snappy. consequently the proposed watermarking plan mulled over PSO is for improve. diverse investigations have been pushed on the proposed manner to manipulate analyzethe execution. The got results display that the proposed system is higher than customary strategies and is the general public of the more capably comfortable over Gaussian confusion, Salt and Pepper Commotion, middle Sifting, Trimming, Pivot, Complexity development, Scaling and Histogram Leveling attacks.
\end{abstract}

Index Terms: Digital image watermarking, IWT, DCT, SVD, GA, PSO, PSNR, NC, SSIM.

\section{INTRODUCTION}

the existing improvement driven economic framework realizes comfort and thought for digitization of the academic trinket. Propelled photograph Watermarking $[1,2]$ is the approach of expansion of picture watermark in media substance and its extraction, on every occasion required, for certification or ownership take a look at of media content. A propelled picture watermark is the records that is covered up authentically in media content material, it is immaterial to a human onlooker [3]. The realness of a computerized substance sensitive watermarking is applied whilst, with the end purpose of copyright assure, healthy watermarking is applied. This request is software-set up.Watermarking plans may be portrayed into outwardly weakened, semi-trance, and non-stupor getting ready. One more is potential relying at the hole of embedding of watermark: spatial and repeat. a specific look at of watermarking plans can be determined in $[4,5]$. In programmed image watermarking, streamlining placing in constant to such an amount, that the watermarked image need to be possible and unpretentious freed from situation. commonly, the individual made mental aptitude approaches, as an instance, Hereditary figuring (GA), Molecule swarm streamlining (PSO), Subterranean insect territory improvement (ACO, and so on., systems might be

Revised Manuscript Received on 16 September, 2019.

CH. Ravi Kumar, Research Scholar, Dept. of ECE, AU College of Engineering, Visakhapatnam, India.

(email: ravikumarsan@gmail.com)

Dr.R. Surya PrakasaRao, Assistant Professor, Dept.of ECE, GVPCE

(A), Visakhapatnam, India.

(email: suryaprakash@gvpce.ac.in)

Dr. P. Rajesh Kumar, Professor \&BOS Chairman, Dept. of ECE, AU College of Engineering, Visakhapatnam, India.

(email: rajeshauce@gmail.com) used for headway capacities. In [6], by way of joining the every GA and PSO to get a sophisticated display .anyways, [6] breaks down the photograph through the maximum severe usually found out Discrete wavelet substitute (DWT) this is having simple difficulty of insights setback. In mild of the down investigating framework in DWT,,the person of the watermark is probably decreased. In [7], a square plan watermarking is proposed based totally really as for Discrete Wavelet change (DWT) and particular well worth breaking down (DWT. The estimation band ended up supplied to SVD from the were given sub agencies. In light of GA,PSO method joins the host photo institutionalization and techniques for dealing with into squares to restrict upset and scaling assaults. At that aspect IWT is achieved on each square and the have been given wager band is discovered to institutionalized SVD. Embedding. The display emerge as evaluated for explicit assortments of moves. The rest of the paper is looked after out as seeks after: phase II outlines the nuances of associated paintings. portion III gives a have a look at more or less the starter musings used on this paper. Territory IV strains the nuances of proposed strategy. Fragment V diagrams execution appraisal nuances at last region VI shuts the paper.

\section{RELATED WORK}

In a robust picture watermarking plan investigators have accomplished in spatial and changed area that find out a replacement off amongst those targets. The spatial spot watermarking strategies truely embed with the manual of fixing the pixel regards in the watermark into the host image [8-11]. those methodologies for the maximum intense element are less floor-breaking to photo and banner adapting to ambushes and required low computational undertakings there are numerous trade territory watermarking frameworks, for instance, Discrete cosine changes (DCT) [12], discrete Fourier changes (DFT) [13-14], discrete wavelet adjustments (DWT) [15-17], and one among a kind nicely worth crumbling (SVD) $[2,18-20]$. these techniques greater often than no longer deliver higher photo elusiveness The advent of watermarking strategies changed into additionally advanced by using uniting in any event alterations [21-33]. The singular nicely worth rot (SVD) is extensively implemented in image watermarking discipline. anyhow, unmistakable masters alluded to as regard for the manufactured super person trouble in SVD-fundamentally primarily based computations [7, 34-35]. Various 
researchers have proposed advanced types of SVD-based totally actually photograph watermarking plans. A compelling photograph watermarking plan contingent upon SVD that embeds the full watermark is given in [20]-The vagary of a picture watermarking plan utilising rectangular mainly based totally SVD proposed in [19] is advanced by means of becoming a member of pay diversion. features, so a couple of visible fashions, as an instance, human substantive structure (HVS) can be combined in locating the right putting dependent on, an outwardly debilitated SVDprincipally based watermarking plan is proven in [36]. The host photograph is segmented into non-masking squares then the putting squares are picked problem to the whole of visible and facet entropies. The watermark bits are hooked up through using converting the coefficients in the preeminent area of the left single vector pass section of the intention squares.. It has ventured forward the relentless high quality of the watermarking yet surrendered the straightforwardness. In like manner, those plans are affordable handiest for the specifically differentiating watermark. A watermarking plan proposed in [32] depends upon on the SVD subspace can defend diverse realities about a image. along these follows, it embeds the important region, enlargement of left particular vector community On a similar idea, Run et al. [33] supplied a picture watermarking plan embedding the essential a chunk of the watermark in repeat area (DCT and DWT areas, resp.). also, an development method is actualized to get a appropriate scaling additives for putting in. in spite of the way that the functions remoted are healthy for such big portions of strikes, the viability of watermarking approach in like way depends upon the watermarking constant. PSO is a intelligent computation that the usage of the stochastic, populace essentially based laptop estimation for important addressing. Zheng [37] achieved the PSO to leaf through the embedding location of the overall range DCT coefficients in a rectangular to improve the crucial of vagary and energy in watermarking. Vahedi [38] utilized the PSO strategy to check for the appropriate essentialness of setting watermark to modify the acceptable and energy of watermarked image. In [39], a watermarking technique modified into proposed relying upon Hereditary Calculation (GA). In [23], GA changed into used for the decision of watermarking relentless. beginning past due, particle swarm improve (PSO) modified into unrivaled into the watermarking shape. Hai Tao [40] implemented PSO for the advancement of scaling factors PSO for development. no matter the way that the PSO became implemented, there might be a nonrecoverable measurements incident because of the 3diploma DWT. In [41], a solitary watermarking moved towards becoming proposed with the guide of PSO as a streamlining operator and whole number wavelet exchange as an alternate technique. brushing off the manner that the realities incident become diminished in [41], there watched a dwindled vitality in particular attacks like disillusioned, histogram alternate and cutting.

\section{PRLEMINARIES}

\section{A. complete assortment Wavelet alternate (IWT)}

The in productivity with wavelet change is its incapacity to carry down the absence of facts in the primary photograph. as an instance, if any of the square of particular photograph having complete range pixel regards and changed via a floating point wavelet trade. The truncation of drifting factor regards will result in absence of certainties, To avoid this trouble, an invertible complete range toquantity wavelet alternate contingent upon lifting [41] is implemented inside the proposed arrangement. It maps complete numbers to complete numbers and doesn't motive any loss of information through ahead and in reverse alternative.

The in advance exchange of an ordinary lifting plan more noteworthy always than no longer consists of of 3 stages: split, estimate and supplant. take into account a sign: $\mathrm{X}=$ $\{x(n), n \in Z\}$ with $x(n) \in R$. The implementation of the forward transform is illustrated as below:

(1) Split: The original signal $X$ is split into two subsets: even indexed samples $x_{e}$ and odd indexed sample $x_{o}$ by means of a sample operation:

$$
\left\{\begin{array}{c}
x_{e}=x(2 n) \\
x_{o}=x(2 n+1)
\end{array}\right.
$$

(1)

After the split operation is completed, the odd set and even set are obtained and the two sets are closely correlated.

(2) Prediction: Given the odd indexed samples $x_{o}$, a predictor $P$ for the even indexed samples $x_{e}$ can be designed:

$$
\widetilde{x_{o}}=P\left(x_{e}\right)
$$

The difference denoted as $d$ between the predicted results and the odd samples is considered as the detail coefficients of the signal $x(n)$, and it is expressed as:

$$
d=x_{o}-\widetilde{x_{o}}=x_{o}-P\left(x_{e}\right)
$$

(3) Update: Knowing the even sample $e x$ and the detail coefficients $d$, the approximation coefficients $c$ are calculated using the updating operator $U$ as:

$$
c=x_{e}+U(d)
$$

The inverse transform can immediately be derived from the forward transform by running the lifting scheme backwards. The block diagram of the lifting scheme is given in Figure 1.

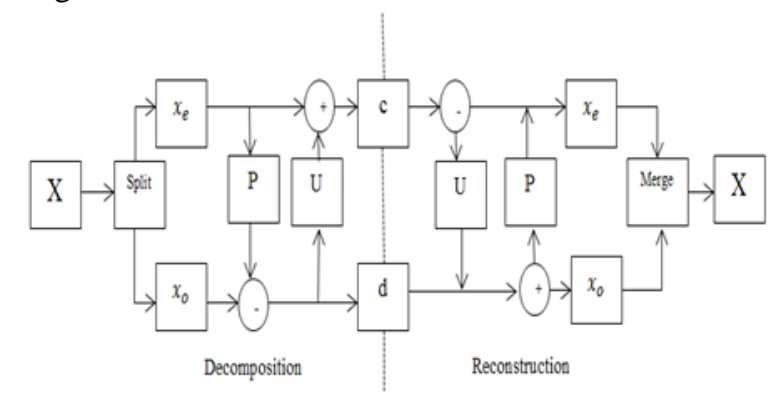

\section{B. Singular Value Decomposition (SVD)}

The singular value decomposition (SVD) [20] is a numerical analysis technique based on a theorem of linear algebra that decomposes a rectangular matrix into the 
product of three matrices: an orthogonal matrix $(U)$, a diagonal matrix $(S)$, and the transpose of an orthogonal matrix $(V)$. It may be considered as a method of transforming correlated data set into uncorrelated one that better explains the various relationships among the original data. represented mathematically as

$A=U S V^{T}$,

where $U U^{T}=I_{m}$ and $V V^{T}=I_{n}$; the columns of $U$ are ortho normal eigenvectors of $A A^{T}$, the columns of $V$ are ortho normal vectors of $A^{T} A$, and $S$ is a diagonal matrix containing the square roots of the eigen values from $U$ or $V$ in descending order. If $r(r \leq n)$ is the rank of the matrix $A$.the diagonal matrix $S$ satisfy the relation (6) and the matrix $A$ can be written as (7):

$$
\lambda_{1} \geq \lambda_{2} \geq \cdots \geq \lambda_{r}>\lambda_{r+1}=\lambda_{r+2} \cdots=\lambda_{n}=0,
$$$$
A=\sum_{k=1}^{r} \lambda_{k} u_{k} v_{k}^{T}
$$

Where $u_{k}$ and $\mathrm{V}_{k}$ are the $k_{\text {th }}$ eigenvector of $U$ and $V$ and $\lambda_{k}$ is the $k_{\text {th }}$ singular value.

\section{DCT}

Discrete cosine transform (DCT) is popular rework technique which transforms a sign from spatial domain to frequency domain and it has notable power compaction belongings.

D. Particle Swarm Optimization (PSO)

PSO [40] is encouraged from the social conduct of organism along with chicken flocking or fish education. Like other evolutionary algorithms the location of a particle is laid low with each the best function visited through it and the position of the pleasant particle in its community. The high-quality particle in the population is denoted through international nice (gbest), while the satisfactory position that has been visited by using the present day particle is denoted through local satisfactory (pbest).

$$
\begin{aligned}
& v_{i}(n+1)=w_{i} v_{i}+c_{1} \operatorname{rand}_{1}\left(\text { pbest }-x_{i}(n)\right)+ \\
& c_{2} \operatorname{rand}_{2}\left(\text { gbest }_{-}-x_{i}(n)\right) \\
& x_{i}(n+1)=x_{i}(n)+v_{i}(n+1) \\
& \quad \text { Where: }
\end{aligned}
$$

$x_{i}(n+1)$ and $x_{i}(n)$ represent the current and the previous positions of particle $\mathrm{i}$

$v_{i}(n+1)$ and $v_{i}(n)$ are the current and the previous velocity of the particle $i$.

rand $_{1}$ andrand ${ }_{2}$ are random numbers uniformly distributed within $[0,1]$.

$W$ is an inertia weight which controls the momentum of the particle.

Commonly the inertia weight is set according to the following equation:

$$
w_{i}=w_{\max }-\frac{w_{\max }-w_{\min }}{\text { iter }_{\max }} . \text { iter }
$$

Where: iter $_{\text {max }}$ is the maximum number of iterations, and iteris the current number of iterations. Each particle in PSO shares the information with its neighbors. The updating equations (4) and (5) combine both of the cognition component

\section{PROPOSED WATERMARKING SCHEME}

The proposed approach [14] is having two phases named as embedding phase and extracting phase. The block diagrams for embedding and extracting phases are as shown in figure 2 and 3respectively.

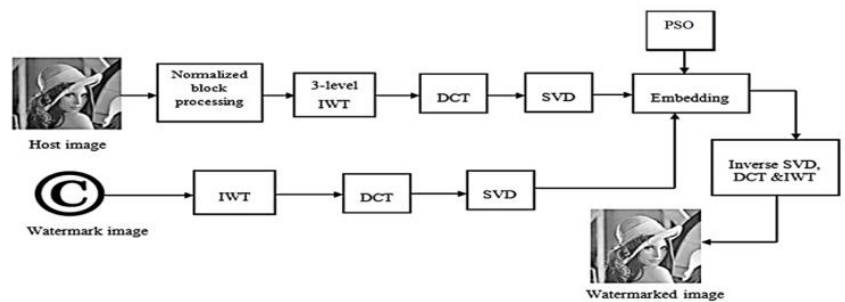

Figure 2: Block diagram of embedding Unit for NBPIWT-DCT-SVD-PSO

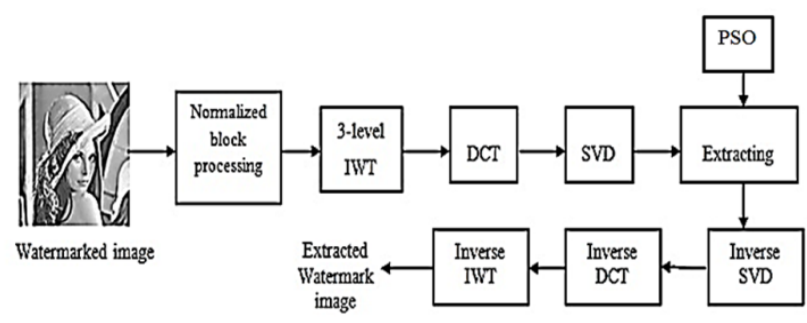

Figure 3. Block diagram of extracting Unit for NBPIWT-DCT-SVD-PSO

\section{A. Embedding Procedure}

Step 1: Let $\mathrm{W}$ be the watermark image of size $\mathrm{KxL}$. Perform one level IWT on the Watermark image W followed by DCT.

$\left\{W_{L L}, W_{L H}, W_{H L}, W_{H H}\right\}=\operatorname{lwt} 2(W)$

Step 2: Apply SVD over the obtained all bands $\left[u_{L L}^{w} s_{L L}^{w} v_{L L}^{w}\right]=\operatorname{svd}\left(W_{L L}\right)$,

$\left[u_{L H}^{w} s_{L H}^{w} v_{L H}^{w}\right]=\operatorname{svd}\left(W_{L H}\right)$,

$\left[u_{H L}^{w} s_{H L}^{w} v_{H L}^{w}\right]=\operatorname{svd}\left(W_{H L}\right)$,

$\left[u_{H H}^{w} s_{H H}^{w} v_{H H}^{w}\right]=\operatorname{svd}\left(W_{H H}\right)$

Step 3: The watermark principal component is obtained by multiplying the matrices $s_{i}^{w}$ and $u_{i}^{w}, \quad W_{i}^{u s}=u_{i}^{w} * s_{i}^{w}$

Step 4: Let $\mathrm{H}$ be original host image of size $M x N$ and perform 3-level IWT over $\mathrm{H}$ followed by DCT.

$\left\{H_{L L}^{1}, H_{L H}^{1}, H_{H L}^{1}, H_{H H}^{1}\right\}=l w t 2(H),\left\{H_{L L}^{2}, H_{L H}^{2}, H_{H L}^{2}, H_{H H}^{2}\right\}=$ $\operatorname{lwt} 2\left(H_{L L}^{1}\right)$

$\left\{H_{L L}^{3}, H_{L H}^{3}, H_{H L}^{3}, H_{H H}^{3}\right\}=l w t 2\left(H_{L L}^{2}\right)$

Step 5: Perform SVD over the obtained all bands at third level of decomposition such as only on $H_{L L}^{3}, H_{L H}^{3}, H_{H L}^{3}, H_{H H}^{3}$.

$\left[u_{L L}^{H} s_{L L}^{H} v_{L L}^{H}\right]=\operatorname{svd}\left(H_{L L}^{3}\right)$

$\left[u_{L H}^{H} s_{L H}^{H} v_{L H}^{H}\right]=\operatorname{svd}\left(H_{L H}^{3}\right)$,

$\left[u_{H L}^{H} s_{H L}^{H} v_{H L}^{H}\right]=\operatorname{svd}\left(H_{H L}^{3}\right)$,

$\left[u_{H H}^{H} s_{H H}^{H} v_{H H}^{H}\right]=\operatorname{svd}\left(H_{H H}^{3}\right)$

Step 6:Change the singular values of host image by embedding the singular value of watermark in all bands $\operatorname{ass}_{i}^{*}=s_{i}^{H}+\alpha * W_{i}^{u s}$

Where

$i \rightarrow\{L L, L H, H L, H H\}$ and $s_{i}^{*}$ is the modified singular value for each band.

Step 7: Apply the inverse SVD on the modified singular values and the obtained modified bands are represented as 
$W M_{i}^{3}=u_{i}^{H} * S_{i}^{*} *\left(v_{i}^{H}\right)^{T}, W M_{L H}^{3}, W M_{H L}^{3}, W M_{H H}^{3}$

Where, $W M_{i}^{3}$ is the modified $i^{\text {th }}$ band,

$i \rightarrow\{L L, L H, H L, H H\}$.

Step 8: Perform Inverse IWT over the obtained modified bands to get the watermarked image (WM) after Inverse DCT.

Step 9: Perform Inverse IWT over the obtained modified bands to get the watermarked image (WM) after Inverse DCT.

$$
\begin{aligned}
& W M_{L L}^{2}=i l w t 2\left(W M_{L L}^{3}, W M_{L H}^{3}, W M_{H L}^{3}, W M_{H H}^{3}\right) \\
& W M_{L L}^{1}=i l w t 2\left(W M_{L L}^{2}, H_{L H}^{2}, H_{H L}^{2}, H_{H H}^{2}\right) \\
& W M=i l w t 2\left(W M_{L L}^{1}, H_{L H}^{1}, H_{H L}^{1}, H_{H H}^{1}\right)(20)
\end{aligned}
$$

Step 10: Apply various attacks on the watermarked image.

\section{B. Extraction Procedure}

Step 1: Apply the normalized block processing on the distorted watermarked image $\left(W M^{*}\right)$ to obtain the distorted normalized watermarked image $\left(N_{W M}^{*}\right)$.

Step 2:Apply 3-level IWTon the distorted \& normalized watermarked image $\left(N_{W M}^{*}\right)$ followed by DCT,

$\left\{\left(N_{W M}^{*}\right)_{L L}^{1},\left(N_{W M}^{*}\right)_{L H}^{1},\left(N_{W M}^{*}\right)_{H L}^{1},\left(N_{W M}^{*}\right)_{H H}^{1}\right\}=$ $\operatorname{lwt} 2\left(N_{W M}^{*}\right)(21)$

$\left\{\left(N_{W M}^{*}\right)_{L L}^{2},\left(N_{W M}^{*}\right)_{L H}^{2},\left(N_{W M}^{*}\right)_{H L}^{2},\left(N_{W M}^{*}\right)_{H H}^{2}\right\}=$ $\operatorname{lwt} 2\left(\left(N_{W M}^{*}\right)_{L L}^{1}\right)(22)$

$\left\{\left(N_{W M}^{*}\right)_{L L}^{3},\left(N_{W M}^{*}\right)_{L H}^{3},\left(N_{W M}^{*}\right)_{H L}^{3},\left(N_{W M}^{*}\right)_{H H}^{3}\right\}=$ $\operatorname{lwt} 2\left(\left(N_{W M}^{*}\right)_{L L}^{2}\right)$

Step 3:Perform SVD over the obtained all bands at third level of decomposition such as only on $\left(N_{W M}^{*}\right)_{L L}^{3},\left(N_{W M}^{*}\right)_{L H}^{3},\left(N_{W M}^{*}\right)_{H L}^{3},\left(N_{W M}^{*}\right)_{H H}^{3}$.

$\left[u_{L L}^{w m} s_{L L}^{w m} v_{L L}^{w m}\right]=\operatorname{svd}\left(\left(N_{W M}^{*}\right)_{L L}^{3}\right)$, $\operatorname{svd}\left(\left(N_{W M}^{*}\right)_{L H}^{3}\right)$

$\left[u_{H L}^{w m} s_{H L}^{w m} v_{H L}^{w m}\right]=\operatorname{svd}\left(\left(N_{W M}^{*}\right)_{H L}^{3}\right), \quad\left[u_{H H}^{w m} s_{H H}^{w m} v_{H H}^{w m}\right]=$ $\operatorname{svd}\left(\left(N_{W M}^{*}\right)_{H H}^{3}\right)(24)$

Step 4: Extract the distorted principal components $W_{i}^{u s^{*}}=\frac{\left(s_{i}^{w m}-s_{i}^{H}\right)}{\alpha}, i \in\{L L, L H, H L, H H\}$

Where $W_{i}^{u s^{*}}$ are the distorted principal components of extracted watermark..

Step 5: The distorted bands are obtained as

$W_{i}^{*}=W_{i}^{u s^{*}} *\left(v_{i}^{w}\right)^{T}, i \in\{L L, L H, H L, H H\}$

Step 6: The final watermark can be extracted by applying inverse DCT and IWT on the obtained distorted wavelet sub-bands as

$$
W^{*}=i l w t 2\left(W_{L L}^{*}, W_{L H}^{*}, W_{H L}^{*}, W_{H H}^{*}\right)
$$

\section{V.SIMULATION RESULTS}

This section breaks down the exhibition of a proposed watermarking plan underneath different analyses. assorted investigate pictures are taken for exploratory evaluation.

The figures 3 (an) and 3 (b) are taken as host pictures while the figures 3 (c) and three (d) are taken in light of the fact that the watermark photograph.

the watermarked picture is exposed to attack of fourteen sorts. Standardized connection (NC) coefficient is utilized as a likeness measure between the bona fide and separated watermark photograph. that enables you to legitimize the general execution of the proposed plan, outcomes are in contrast with the conventional technique.

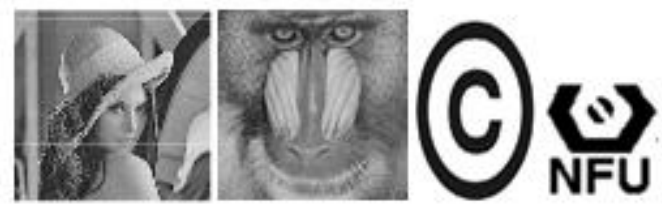

Fig.3(a) 3(b) 3(c) 3(d)

Figure3: (a) Lena host image (b) Baboon host image

(c) Copyright Watermark image (d) NFU logo as watermark image

The performance of the proposed technique is evaluated for the take a look at instances of Lena is taken as host image and Copyright is taken as the watermark photograph [39]. within the second take a look at case the Baboon image is taken as a host and the NFU logo photo is taken as watermark as like in base paper [40]. on this end result, first of all the received visual consequences (watermarked picture and Extracted watermark) for the proposed approach NBP-IWT-DCT-SVD-PSO depicted in theIV , after various attacks applied on watermarked picture.

Table 1: Performance comparative table for the test case of Baboon with NFU logo as watermark

\begin{tabular}{|c|c|c|c|c|}
\hline \multirow{2}{*}{ Attacks } & \multicolumn{2}{|c|}{$\begin{array}{c}\text { Existing Method (SVR- } \\
\text { SVD-PSO) }\end{array}$} & \multicolumn{2}{c|}{$\begin{array}{c}\text { Proposed Method (NBP- } \\
\text { IWT-DCT-SVD-PSO) }\end{array}$} \\
\hline & PSNR & NC & PSNR & NC \\
\hline WN (25\%) & 20.13 & 0.9068 & 41.80 & 0.9321 \\
\hline SPA (3\%) & 20.62 & 0.9137 & 43.26 & 0.9518 \\
\hline U (3\%) & 23.78 & 0.9889 & 40.74 & 0.9814 \\
\hline GNF (3X3) & 35.28 & 0.9615 & 44.30 & 0.9602 \\
\hline MF (3X3) & 33.94 & 0.9388 & 40.98 & 0.9385 \\
\hline AF (3X3) & 28.8 & 0.9461 & 38.90 & 0.9547 \\
\hline SHF (3X3) & 22.2 & 0.9639 & 39.68 & 0.9659 \\
\hline $\begin{array}{c}\text { JPEG } \\
(50 \%)\end{array}$ & 26.93 & 0.9898 & 44.81 & 0.9428 \\
\hline $\begin{array}{c}\text { BRIGHT } \\
(+20)\end{array}$ & 18.69 & 0.9639 & 46.86 & 0.9612 \\
\hline $\begin{array}{c}\text { DARKEN } \\
(-20)\end{array}$ & 18.55 & 0.9409 & 45.99 & 0.9608 \\
\hline HE & 16.99 & 0.9105 & 42.31 & 0.9501 \\
\hline SA (25\%) & 33.72 & 0.9830 & 40.09 & 0.9615 \\
\hline CA (25\%) & 13.38 & 0.8992 & 38.25 & 0.9481 \\
\hline $\begin{array}{c}\text { PAINTING } \\
(\text { NFU) }\end{array}$ & 13.26 & 0.9207 & 39.97 & 0.9516 \\
\hline
\end{tabular}


Table 2: Performance metrics for the proposed methods (Lena with copyright image as watermark)

\begin{tabular}{|l|l|l|l|l|}
\hline \multirow{2}{*}{ Attacks } & \multicolumn{2}{|l|}{$\begin{array}{l}\text { Proposed } \\
\text { NBP-IWT-DCThod Using }\end{array}$} & $\begin{array}{l}\text { Proposed } \\
\text { NBP-IWT-DCT-SVD-PSO }\end{array}$ \\
\hline & PSNR & NC & PSNR & NC \\
\hline NA & 44.18 & 0.9529 & 46.89 & 0.9692 \\
\hline GNA (0.01) & 42.09 & 0.9441 & 43.67 & 0.9590 \\
\hline SPA (0.05) & 41.26 & 0.9381 & 42.85 & 0.9482 \\
\hline MFA (3X3) & 39.86 & 0.9237 & 41.02 & 0.9403 \\
\hline AFA (3X3) & 37.28 & 0.9210 & 38.94 & 0.9489 \\
\hline HEA & 39.51 & 0.9229 & 41.26 & 0.9465 \\
\hline CEA & 37.90 & 0.9106 & 38.79 & 0.9612 \\
\hline JCA (50\%) & 42.19 & 0.9268 & 44.23 & 0.9362 \\
\hline RA (50\%) & 39.26 & 0.9297 & 41.38 & 0.9368 \\
\hline RSA (50\%) & 41.78 & 0.9292 & 42.78 & 0.9390 \\
\hline CA (35\%) & 34.82 & 0.8916 & 34.35 & 0.9025 \\
\hline FRA & 39.24 & 0.8998 & 41.26 & 0.9025 \\
\hline FCA & 41.68 & 0.9010 & 42.07 & 0.9162 \\
\hline TA (40X40) & 41.03 & 0.8991 & 41.63 & 0.9135 \\
\hline
\end{tabular}

Table. 1 represents Performance comparative table for the test case of Baboon with NFU logo as watermark.The attacks are compared proposed(NBP-IWT-DCT-SVDPSO)with Existing Method (SVR-SVD-PSO).Table.2 represents Performance metrics for the proposed methods (Lena with copyright image as watermark.The attacks are compared proposedMethod Using NBP-IWT-DCT-SVDPSO with Existing Method(NBP-IWT-DCT-SVD-GA).

Table. 3 represents obtained results for the test case of Baboon with NFU logo as watermark.The attacks are compared proposed(NBP-IWT-DCT-SVD-PSO)with Existing Method (SVR-SVD-PSO).

Table 3: Obtained visual Results for the proposed NBP-IWT-DCT-SVD-PSO

\begin{tabular}{|c|c|c|c|c|c|}
\hline Attack & $\begin{array}{c}\text { Watermarke } \\
\text { d Image }\end{array}$ & \begin{tabular}{|c|} 
Extracted \\
Watermark \\
\end{tabular} & Attack & $\begin{array}{c}\text { Watermarked } \\
\text { Image } \\
\end{array}$ & $\begin{array}{c}\text { Extracted } \\
\text { Watermark }\end{array}$ \\
\hline $\mathrm{NA}$ & $\left.\right|_{\text {PSNR: } 46.89}$ & & $\mathrm{JCA}$ & ${ }_{\text {PSNR: } 4423}$ & $\underbrace{}_{N C: 0.9362}$ \\
\hline GNA & & & $R A$ & 1.38 & \\
\hline SPA & $\frac{181}{\text { PSNR } 42.85}$ & & RSA & & \\
\hline SNA & $\frac{16}{\text { PSNR:43.76 }}$ & 369 & $\mathrm{CA}$ & & \\
\hline MFA & $\frac{\|_{\text {PSNR:41.02 }}}{4}$ & NC & FRA & PSNR 41.26 & \\
\hline AFA & ${ }_{\text {PSNR: } 3894}$ & 9439 & FCA & PSNB & NC:0.9162 \\
\hline HEA & PSNR:41.26 & NC: 09465 & $\mathrm{TA}$ & & 35 \\
\hline CEA & $\int_{\text {PSNR } 38.79}$ & $\frac{(\mathrm{C})}{\text { NC:09612 }}$ & & & \\
\hline
\end{tabular}

\section{CONCLUSION}

This paper has delineated the proposed NBP-IWT-DCTSVD-PSO. because the wager band passes on regularly substantial statistics, yet extra prominent defilement inside the concept of watermarked photo and along those traces it is actualized to leftover organizations seperately. From the era effects, the proper watermarking steady for LL band and for terrific corporations is noticeable to be zero.05 and $0.1 / 2$ as I would like to suppose. numerous multiplications are completed over the proposed technique thru fluctuating host and watermark previews. in this imitation, it will in popular be gathered that the proposed system outmaneuvers the past proposed technique(NBP-IWT-DCT-SVD-GA) and everyday philosophy. On a commonplace the proposed strategy finished an increase of $2.0117 \mathrm{~dB}$ in PSNR and 0.013 in $\mathrm{NC}$ for the trial of Lena with Copyright over the proposed technique the usage of NBP-IWT-DCT-SVD-GA.

From a comparable assessment of desk 1; it's far visible that the proposed technique were given an expansion of $18.695 \mathrm{~dB}$ in PSNR and 0.0095 in NC for the evaluation of Monkey with NFU logo over the proposed method the usage of NBP-IWT-DCT-SVD-PSO.

\section{REFERENCES}

1 I. J. Cox, J. Kilian, F. T. Leighton, and T. Shamoon, "comfortable unfurl collection watermarking for sight and sound," IEEE Exchanges on photograph getting ready, vol. 6, no. 12, pp. 1673-1687, 1997.

2 R. Liu and T. Tan, "A SVD-primarily based watermarking plan for verifying genuine ownership," IEEE Exchanges on Media, vol. 4, no. 1, pp. 121-128, 2002.

3 M. L. manufacturing facility administrator, I. J. Cox, J. P. M. G. Linnartz, and T. Kalker, "A evaluate of watermarking requirements and practices," in advanced sign getting ready in Sight and sound Frameworks, pp. 461-485, 1999.

4 E. Hussein and M. A. Belal, "automatic watermarking techniques, applications and ambushes applied to major media: an outline," worldwide Diary of Designing Exploration and Innovation, vol. 1, no. 7, pp. 1-eight, 2012.

5 Khan, A. Siddiqa, S.Munib, and S. A.Malik, "An continuous investigate of reversible watermarking tactics," records Sciences, vol. 279, pp. 251-272, 2014.

6 L.- D. Li and B.- L. Guo, "restrained picture watermarking in spatial area impenetrable to geometric attacks," AEU: worldwide Diary of gadgets and Correspondences, vol. Sixty 3, no. 2, pp. 123-131, 2009.

7 Q. Su, Y. Niu, Q. Wang, and G. Sheng, "A outwardly debilitated concealing picture watermarking reliant on DC aspect within the spatial area," Optik, vol. 124, no. 23, pp. 6255-6260, 2013.

8 C.- H. Yang, C.- Y. Weng, S.- J. Wang, and H.- M. solar, "flexible statistics stowing without end in side zones of snap shots with spatial LSB area frameworks," IEEE Exchanges on data Crime scene investigation and safety, vol. 3, no. 3, pp. 488-497, 2008.

9 S. S. Jamal, T. Shah, and that i. Hussain, "An groundbreaking plan for programmed watermarking the usage of scattered manual," Nonlinear elements, vol. seventy three, no. 3, pp. 1469-1474, 2013. 
10 S. D. Lin, S.- C. Shie, and J. Y. Guo, "enhancing the intensity of DCT-primarily based image watermarking opposite to JPEG stress," laptop Gauges and Interfaces, vol. 32 , no. 1-2, pp. 54-60, 2010.

11 B. Chen, G. Coatrieux, G. Chen, X. solar, J. L. Coatrieux, and $\mathrm{H}$. Shu, "complete four-D quaternion discrete Fourier alternate primarily based watermarking for concealing photographs," computerized signal preparing, vol. 28, no. 1, pp. 106-119, 2014.

12 J. Lang and Z.- G. Zhang, "Dazzle progressed watermarking technique in the incomplete Fourier trade region," Optics and Lasers in building, vol. Fifty three, pp. 112-121, 2014.

13 E. H. Elshazly, O. S. Faragallah, A. M. Abbas, "full of life and cozy midway wavelet picture watermarking," signal, photograph and Video dealing with, Vol.nine, pp.89-98, December 2015.

14 L. Li, H.- H. Xu, C.- C. Chang, and Y.- Y. mother, "A story picture watermarking in redistributed invariant wavelet space," Diary of Frameworks and Programming, vol. 80 four, no. 6, pp. 923-929, 2011.

15 N. Liu,H. Li, H. Dai, D. Guo, andD.Chen, "energetic outwardly impeded photograph watermarking depending upon wild blends," Nonlinear elements, vol. eighty, no. 3, pp. 1329-1355, 2015.

16 M.- Q. Fan, H.- X. Wang, and S.- okay. Li, "Restudy on SVD-based watermarking plan," carried out science and Calculation, vol. 203, no. 2, pp. 926-930, 2008.

17 A. A.Mohammad, A. Alhaj, and S. Shaltaf, "An advanced SVD essentially based totally watermarking plan for ensuring legitimate proprietorship," sign managing, vol. 88, no. nine, pp. 2158-2180, 2008.

18 Q. Su, Y. Niu, H. Zou, and X. Liu, "A outwardly weakened twofold concealing snap shots watermarking depending upon singular well well worth decay," implemented technological know-how and Calculation, vol. 219 , no. 16 , pp. 8455-8466, 2013.

19 H.- T. Hu and L.- Y. Hsu, "Investigating DWT-SVDDCT spotlight parameters for wholesome exceptional watermarking towards JPEG and JPEG2000 stress," computers and electrical constructing, vol. forty one, pp. Fifty two-sixty three, 2015.

20 N. M.Makbol and B. E. Khoo, "Hearty outwardly weakened image watermarking plan contingent upon Repetitive Discrete Wavelet change and precise really worth Decomposition," AEU-common Diary of hardware and Correspondences, vol. 67,no. 2, pp. 102112, 2013.

21 N. M.Makbol and B. E. Khoo, "each other extremely good and cozy mechanized image watermarking plan contingent upon the whole extensive assortment wavelet alternate and singular well well worth deterioration," computerized sign preparing, vol. 33, pp. 134-147, 2014.

$22 \mathrm{X}$. Wu and W. solar, "Hearty copyright inclusion plot for reducing edge snap shots utilizing covering DCT and SVD," carried out sensitive Registering Diary, vol. thirteen, no. 2, pp. 1170-1182, 2013.

23 P.- P. Zheng, J. Feng, Z. Li, and M.- Q. Zhou, "A story SVD and LS-SVM mix figuring for outwardly disabled watermarking," Neurocomputing, vol. 142, pp. 520-528, 2014.

24 A. Mishra, C. Agarwal, A. Sharma, and P. Bedi, "Upgraded diminish scale photo watermarking utilising DWT-SVD and Firefly algorithm," master Frameworks with applications, vol. 41, no. 17, pp. 7858-7867, 2014.

25 M. Ali and C.W. Ahn, "An overhauled watermarking method reliant on self-adaptable de in DWT-SVD alternate area," signal managing, vol. ninety 4, no. 1, pp. 545-556, 2014.

26 M. Ali, C. W. Ahn, and M. Pant, "A successful picture watermarking approach utilising SVD and differential advancement in DCT area," Optik, vol. 100 twenty five, no. 1, pp. 428-434, 2014.

27 M. Ali, C.W. Ahn, M. Wheeze, and P. Siarry, "A photograph watermarking plan in wavelet location with cutting facet pay of singular nicely really worth breaking down with the aid of faux bumble bee province," data Sciences, vol. 301, pp. 44-60, 2015.

28 M. Ali, C. W. Ahn, and P. Siarry, "Differential development figuring for the selection of ideal scaling factors in picture watermarking," constructing uses of automatic reasoning, vol. 31, pp. 15-26, 2014.

29 S. H. Amiri and M. Jamzad, "lively watermarking contrary to print and compass strike through awesome displaying calculation," signal dealing with: photo Correspondence, vol. 29, no. 10, pp. 1181-1196, 2014.

30 B. Lei, D. Ni, S. Chen, T. Wang, and F. Zhou, "perfect photograph watermarking plan reliant on cluttered guide and quaternion wavelet trade," Nonlinear factors, vol. 78, no. four, pp. 2897-2907, 2014.

31 R.- S. Run, S.- J. Horng, J.- L. Lai, T.- W. Kao, and R.- J. Chen, "An advanced SVD-primarily based certainly watermarking framework for copyright warranty," grasp Frameworks with programs, vol. 39, no. 1, pp. 673-689, 2012.

32 M. Ali and C. W. Ahn, "feedback on 'upgraded dull scale photograph watermarking the usage of DWT-SVD and Firefly set of rules'," grasp Frameworks with programs, vol. 40 two, no. 5, pp. 2392-2394, 2015.

33 J.- M.Guo and H. Prasetyo, "protection examinations of the watermarking plan contingent upon tedious discrete wavelet trade and precise well worth deterioration," AEU—global Diary of devices

34 C.- C. Lai, "An improved SVD-based totally in reality watermarking plan the use of human visible attributes," Optics Correspondences, vol. 284, no. 4, pp. 938-944, 2011.

35 Zheng, Y., Wu, C. H., Lu, Z. M., \&Ip, W. H. "best beneficiant picture watermarking situation to PSO and HVS in wide collection DCT location", global Diary of computer Sciences and constructing Framework, Vol.2, No.4, pp.281-287, 2008.

36 Vahedi, E., Lucas, C., Zoroofi, R. An., and Shiva, M, "some different technique for photograph watermarking with the manual of using atom swarm enhancement", In approaches of IEEE ICSPC, 2007, pp. 1383-1386.

37 R. Surya PrakasaRao, Dr. P. Rajesh Kumar, "An proficient Hereditary Calculation primarily based Dim scale computerized image watermarking for enhancing the power and Imperceptibility", In Proc., of accepted assembly on electric, gadgets, and development strategies (ICEEOT), 2016.

38 Hai Tao, JasniMohamadZain, "A wavelet-primarily based particle swarm development be counted for computerized image watermarking", integrated laptop Helped building, VOl.19, pp.81-91, 2012.

39 CH. Ravi Kumar, R. Surya PrakasaRao, Dr. P. Rajesh Kumar, "GA based Lossless and vigorous photo Watermarking the use of NBP-IWT-DCT-SVD Transforms", JARDCS, 2018

40 Hung-Hsu Tsaia, Yu-JieJhuanga, Yen-Shou Lai "A SVD-based absolutely image watermarking in wavelet territory making use of SVR and PSO", applied fragile preparing, 2012. 